\title{
Conclusion Should Shun the Cloud of Confusion
}

\author{
Debajyoti Mohanty $^{1}$ (1) $\cdot$ Dharmendra Dugar $^{1}$
}

Received: 10 April 2021 / Accepted: 24 November 2021 / Published online: 27 November 2021

(c) Association of Surgeons of India 2021

Sir, we have read the article "Randomized control clinical trial of overnight fasting to clear fluid feeding 2 hours prior anesthesia and surgery" by Joshi et al. with interest [1]. This study was planned with the premise that a short interval of preoperative fasting will be beneficial to the patients undergoing elective surgical intervention under general anesthesia (GA). The primary objective of the study was to measure the volume of gastric contents by transabdominal ultrasonogram just prior to intubation. Measuring the $\mathrm{pH}$ of gastric contents obtained through nasogastric aspiration immediate post intubation was the secondary objective. The primary and secondary objectives are essential for formulating the null and the alternate hypothesis of any clinical trial. Both the hypotheses should contradict each other, i.e., if the null hypothesis suggests that the test and control groups have similar outcome then the alternate hypothesis should propose a difference in outcome between the two groups. The hypothesis statements in this study appears perplexing as both the null and the alternate hypothesis are similar in terms of finding "no difference in the value of gastric $\mathrm{pH}^{\mathrm{H}}$ " in the test and the control groups.

The sample size of this study was calculated at 22.94 patients for each group. Calculation of sample size takes into consideration the primary outcome of interest of the study, type I error $(\alpha)$ set at 0.05 , type II error $(\beta)$ set at 0.2 , and power of the study set at $80 \%$. Power of a study indicates the chance of detecting any subtle difference between the two intervention groups. Higher the power of a study, larger is the sample size. The power of this study was variably mentioned at $80 \%$ and $90 \%$ creating confusion regarding the calculation of appropriate sample size. The authors also need to clarify the reason behind their expectation of mean gastric volume less than $27.48 \pm 2$ in estimation of sample size. The article structure lacks a formal "Results" section

Debajyoti Mohanty

debajyoti.mohanty@gmail.com

1 Department of General Surgery, All India Institute

of Medical Sciences, Raipur, Chhattisgarh, India deviating from the standard introduction, methods, results, and discussion (IMRAD) pattern, and all the study observations were clubbed with the discussion [2].

The study concluded that $2 \mathrm{~h}$ fasting prior to elective nonabdominal surgeries under GA had a better outcome in terms of reduced gastric volume and gastric content $\mathrm{pH}$ than the routine practice of overnight fasting. The first conclusion was supported by the statistically significant reduced gastric volume ( $p=<00001$ ) in the $2 \mathrm{~h}$ fasting group than the conventional overnight fasting patients. In contrast, the second conclusion appears debatable as the authors were unable to find statistically significant difference in the mean gastric aspirate $\mathrm{pH}$ in both the groups $(p=<0.12)$. The authors reported small sample size and participation by health volunteers as the limitations. We believe that subjecting healthy individuals to surgical intervention under GA for the sake of the study can raise serious ethical implications and needs to be properly justified.

\section{Declarations}

Conflict of Interest The authors declare no competing interests.

\section{References}

1. Joshi Y, Dhamija S (2021) Randomized control clinical trial of overnight fasting to clear fluid feeding 2 hours prior anesthesia and surgery. Indian J Surg 83:248-54

2. Huth EJ (1987) Structured abstracts for papers reporting clinical trials. Ann Internal Med 106(4):626-7

Publisher's Note Springer Nature remains neutral with regard to jurisdictional claims in published maps and institutional affiliations. 